\title{
EL PAPEL DE LA SOCIEDAD CIVIL en la Cumbre de la Tierra: GOBERNANZA COMPLEJA
}

\author{
Martha Isabel Gómez LeE* \\ NiCOlÁs MAXFIELD**
}

\section{Resumen}

El artículo se enfoca en analizar la participación de la sociedad civil en la Cumbre de la Tierra de 1992, con base en las experiencias del Consejo Internacional para la Ciencia, la Unión Internacional para la Conservación de la Naturaleza y los Recursos Naturales, y la Asociación para el Progreso de la Comunicación en la Cumbre de la Tierra. El marco teórico está fundamentado en el trabajo de Andrew Hurrell y busca identificar las actividades de gobernanza compleja que fueron adelantadas por estos actores de la sociedad civil y examinar sus herramientas de influencia. La evidencia encontrada corrobora el potencial de la sociedad civil para influir en la interpretación de asuntos políticos por medio de actividades de gobernanza compleja en la Cumbre de la Tierra. Se concluye que los estudios de la sociedad internacional se deben enfocar en la manera como los Estados interactúan con una serie de actores sociales, como la sociedad civil.

Palabras clave: gobernanza, sociedad civil, Cumbre de la Tierra, comunidades epistémicas.

\section{THE ROLE OF CIVIL SOCIETY IN THE EARTH SUMMIT: COMPLEX GOVERNANCE}

\section{Abstract}

This article focuses on analyzing the participation of civil society in the Earth Summit

* Doctora en Estudios Políticos. Docente investigadora de la Universidad Externado de Colombia, Bogotá (Colombia). [martha.gomez@uexternado.edu.co].

** Magíster en Asuntos Internacionales. Coordinador de movilidad, Universidad Central, Bogotá (Colombia). [nicomaxfield@gmail.com].

Recibido: 15/05/2017/ Modificado: 25/07/2017/ Aceptado: 26/07/2017.

Para citar este artículo

Gómez Lee, M. I. y Maxfield, N. (2017). El papel de la sociedad civil en la Cumbre de la Tierra: gobernanza compleja. OPERA, 21, pp. 135-156.

DoI: https://doi.org/10.18601/16578651.n21.08 
of 1992, based on the experiences of the International Council for Science, the International Union for the Conservation of Nature and Natural Resources, and the Association for the Progress of Communication in the Earth Summit. The theoretical framework is based on the book On Global Order by Andrew Hurrell. It seeks to identify the complex governance-related activities put forth by these civil society actors and to examine the tools of influence available. The evidence identified corroborates the potential of civil society to influence the interpretation of political issues through the use of complex governance activities in the Earth Summit. The conclusions are that any studies of international society must focus on how States interact with the different social actors, such as civil society.

Key words: Governance, civil society, Earth Summit, epistemic communities.

En la Cumbre de la Tierra, los líderes políticos buscaron confrontar lo que hoy en día se conoce como el reto ecológico, es decir, la dificultad de los gobiernos de responder adecuadamente a las amenazas generadas por la degradación de la naturaleza. Andrew Hurrell, autor de On Global Order, presenta tres dimensiones de dicho reto. La primera dice que el sistema de Estado-nación no funciona bien porque no proporciona un marco político viable para el manejo del medio ambiente global. La segunda hace referencia a que hay un número creciente de Estados individuales que son incapaces de proporcionar un orden local para gestionar el medio ambiente dentro de sus fronteras. La tercera considera que el Estado es incapaz de gestionar el medio ambiente. El
Estado-nación está perdiendo su lugar como el principal foco de la lealtad política de las personas, quienes se están identificando con otras organizaciones o entidades que pretenden enfrentar los problemas medioambientales (2007, pp. 216-217). En estas condiciones, es lógico concluir que enfrentar el reto ecológico exige la participación de actores no estatales.

Para proponer una respuesta al reto ecológico y a las amenazas asociadas a la degradación del medio ambiente, la Comisión Mundial sobre el Medio Ambiente y el Desarrollo (Comisión Brundtland) expidió el informe llamado "Nuestro futuro común", conocido como el Informe Brundtland. Este planteó los retos medioambientales que el planeta enfrentaba, tales como el cambio climático, la desertificación del suelo y el agujero en la capa de ozono.

Con este informe en la mano, la Asamblea General de la Organización de las Naciones Unidas (ONU) convocó para 1992 la Cumbre de las Naciones Unidas sobre el Medio Ambiente y el Desarrollo de Rio de Janeiro, Brasil, conocida como la Cumbre de la Tierra. En esta cumbre, la comunidad internacional buscó afrontar los desafíos medioambientales y su relación con el desarrollo, y adoptó una serie de instrumentos internacionales, tales como la Agenda 21 y la Declaración de Rio sobre el Medio Ambiente y el Desarrollo, la Convención Marco de las Naciones Unidas sobre Cambio Climático (CMNUCC) y el Convenio sobre la Diversidad Biológica (CDB).

La participación de la sociedad civil en la Cumbre de la Tierra se debe a las decisiones tomadas en los Comités Preparatorios (PrepCom, por sus siglas en inglés). Ya desde la firma de la Resolución 44/228 por parte de 
la Asamblea General, que anunciaba la Cumbre de la Tierra, los actores de la sociedad civil mostraron mucho interés en participar y se presentaron en el Consejo Económico y Social ${ }^{1}$ (ECOsoc, por sus siglas en inglés). Cuando finalizó el último PrepCom Iv, en abril de 1992, el Ecosoc había acreditado a 1.420 organizaciones de la sociedad civil para que pudieran participar en las actividades de la Cumbre de la Tierra (Princen y Finger 1994, p. 200). La mayoría de estas organizaciones ejercieron su influencia a través de conversaciones en los pasillos cuando las delegaciones caminaban de reunión a reunión (p. 207).

En la Cumbre de la Tierra, la sociedad civil realizó una variedad de actividades como foros, conferencias, seminarios, publicaciones y lobby. En el Foro Global 92 participaron cerca de 25.000 organizaciones de la sociedad civil con una amplia gama de iniciativas incorporadas, y los más de 30 tratados alternativos firmados (Rodríguez-Becerra, 1994, p. 34). Manuel Rodríguez-Becerra, el primer ministro del Medio Ambiente de Colombia, destaca que la participación de la sociedad civil durante la Cumbre de la Tierra fue excepcional en cantidad y nivel de ambición. Diana Pombo (2014), importante ambientalista colombina, subraya que en las preparaciones en Colombia para la Cumbre de la Tierra se reunieron varios grupos de la sociedad civil colombiana y sus reflexiones influenciaron la Constitución Política de 1991.
Para los efectos de este artículo se entiende por sociedad civil ${ }^{2}$ un "dominio y espacio donde [existen] grupos intermediarios autoorganizados que son relativamente independientes de las autoridades públicas y actores privados económicos, los cuales son capaces de emprender una acción colectiva en busca de sus intereses o valores, y actúan políticamente" (Hurrell, 2007, p. 100). Para Hurrell, la sociedad civil es en sí misma un escenario de luchas por el poder, en tanto no es homogénea y está cruzada por desigualdades y contradicciones (Serbin, 2009).

En este contexto vale la pena preguntarse ¿cómo fue en concreto el potencial de la sociedad civil para influir en la interpretación de asuntos políticos durante la Cumbre de la Tierra? Se indaga sobre tres actores emblemáticos, el Consejo Internacional para la Ciencia (ICSU), la Unión Internacional para la Conservación de la Naturaleza y los Recursos Naturales (UICN) y la Asociación para el Progreso de la Comunicación (APC). La hipótesis de trabajo es que estos autores tuvieron el potencial de influir en la interpretación de asuntos políticos en la Cumbre de la Tierra al desarrollar actividades propias de la gobernanza compleja.

El marco teórico que sustenta este análisis se basa en el concepto analítico de gobernanza compleja de Andrew Hurrell (2007), entendido como un proceso de construcción de las instituciones y creación de las reglas capaces de regular los flujos y las transacciones que se

1 ECOSOc se encarga de las relaciones entre la onu y los actores de la sociedad civil que están acreditadas (Consultative Status with ECOSOC and other accreditations 2016, 25 de enero)

2 En el libro La sociedad civil: un tabú en Colombia, Erli Margarita Marín llama la atención sobre la dificultad de definir sociedad civil cuando dice, "hay tantas definiciones como percepciones ideológicas" (2011, p. 26). 
llevan a cabo en un ámbito global. Comprende los procesos que ocurren entre los Estados y alrededor de aquellos, por medio de las redes transnacionales que se relacionan con actores civiles y agentes del mercado (p. 95). A raíz de los preparativos para la Cumbre de la Tierra, este autor expresó la dificultad de una negociación internacional por la dicotomía entre la interdependencia ambiental y la fragmentación de los Estados. Asimismo, advirtió que para esta cumbre los Estados estaban divididos entre los del Norte y los del Sur. Los Estados del Norte se preocuparon por temas como cambio climático y el deterioro de la capa de ozono, mientras que los Estados del Sur estaban preocupados por el fracaso del desarrollo, la desertificación y la contaminación de las aguas (Hurrell, 1992, p. 39).

Este análisis es relevante porque contribuye al desarrollo de la teoría de la gobernanza en el campo de las relaciones internacionales, dada su frecuente contemplación del papel de los actores no estatales en las actividades internacionales, lo cual no ocurre en las teorías tradicionales de relaciones internacionales como el realismo y el liberalismo. Vale la pena considerar a Andrew Hurrell, teórico híbrido que utiliza conceptos de la escuela inglesa y la gobernanza, y proporciona el fundamento científico para la realización de un estudio teórico del papel de la sociedad civil en eventos como la Cumbre de la Tierra.

La aplicación práctica de esta investigación se plasma en la labor de poner a prueba la teoría de Hurrell en relación con actores más específicos como las redes transnacionales de advocacy y las comunidades epistémicas. De esta forma puede servir de modelo para otros estudios académicos o políticos sobre gobernanza global, al ofrecer un complemento al conocimiento académico actual y a la vez profundizar detalladamente en las contribuciones de actores específicos que participaron en la Cumbre de la Tierra.

Desde esta perspectiva, el objetivo general de este artículo es analizar el potencial de la sociedad civil para influir en la interpretación de asuntos políticos por medio de actividades de gobernanza compleja durante la Cumbre de la Tierra. Se pretende identificar las actividades de gobernanza compleja que adelantaron los actores de la sociedad civil en dicha Cumbre, y examinar las herramientas de influencia de los actores de la sociedad civil en la interpretación de los asuntos políticos tratados en esta.

Como metodología de la investigación se utilizó el método cualitativo y se fundamentó en los conceptos analíticos planteados por Hurrell. El corpus de la investigación estuvo conformado por fuentes primarias (entrevistas abiertas, las páginas web de la ONU, los documentos legales que resultaron de la Cumbre de la Tierra, propuestas planteadas por los actores de la sociedad civil encontradas en documentos e informes de la ONU, como el Informe Brundtland que fue muy relevante), fuentes secundarias (libros y artículos científicos y comentarios o análisis de fuentes primarias de la Cumbre) y fuentes terciarias como Nongovernmental organizations in world politics: The construction of global governance de Peter Willetts, que sirvió para identificar algunos de los actores más destacados.

Se entrevistó a tres personajes importantes que estuvieron involucrados en la Cumbre de la Tierra de 1992, quienes dieron cuenta 
de los diferentes valores y perspectivas que marcaron este evento y sirvieron para guiar el tono del artículo. En primer lugar, Diana Carolina Pombo Holguín, asesora del ministro de Medio Ambiente de Colombia, quien interactuó directamente con los miembros de la sociedad civil colombiana durante la Cumbre de la Tierra; en segundo lugar, Manuel Rodríguez-Becerra, el primer ministro del Medio Ambiente de Colombia quien participó en esta cumbre de manera activa, y en tercer lugar, Michael Zammit Cutajar, el primer Secretario Ejecutivo de la CMNUCC durante la cumbre. Las entrevistas fueron realizadas de forma abierta, transcurrieron como una conversación, y fueron transcritas. En cuanto a su abordaje y sistematización, fueron examinadas y ordenadas según las evidencias que probaran la viabilidad de una sociedad internacional que funcione usando mecanismos de gobernanza en la que interactúan actores de la sociedad civil.

Por último, el conjunto de técnicas y procedimientos que se siguieron durante el proceso de investigación se sistematizó de la siguiente manera:

Se examinaron los estudios de caso de Consejo Internacional para la Ciencia (ICsu), la Unión Internacional para la Conservación de la Naturaleza y los Recursos Naturales (UICN) y la Asociación para el Progreso de la Comunicación (APC) para determinar en qué medida tuvieron el potencial de influir en la interpretación de los asuntos políticos por medio de actividades de gobernanza compleja.

En primer lugar, se indagó y argumentó sobre las actividades de gobernanza compleja que adelantaron estos tres actores de la socie- dad civil en la Cumbre de la Tierra, verificando si hubo hallazgos sobre las siguientes actividades propias de la gobernanza compleja según Hurrell (2007):

- Participación en el proceso formal de la creación de normas estableciendo estándares y desarrollo de normas.

- Participación en el proceso social de la aparición de nuevas normas y su inclusión en la agenda internacional.

- Verificación del cumplimiento de compromisos.

En segundo lugar, se indagó y argumentó sobre las herramientas de influencia en la interpretación de los asuntos políticos de estos tres actores de la sociedad civil, verificando si hubo hallazgos en cuanto a las siguientes herramientas propias de la gobernanza compleja según Hurrell (2007):

- Habilidad de aprovechar el conocimiento científico o las ideas causales.

- Capacidad de explotar plataformas institucionales.

- Acceso a los Estados por medio de redes y comunidades políticas.

- Habilidad para participar en actos políticos simbólicos.

El artículo está estructurado en cuatro partes: marco teórico, resultados de la investigación, discusión sobre las actividades de gobernanza compleja, y discusión sobre las herramientas de influencia en la interpretación de los asuntos políticos. 


\section{MARCO TEÓRICO: GOBERNANZA COMPLEJA}

Para empezar, se debe tener en cuenta que para analizar las dinámicas que en la Cumbre de la Tierra mantuvieron el orden en la sociedad internacional son insuficientes los abordajes tradicionales y es necesario ampliar la perspectiva teórica, como lo hace Hurrell con su apuesta de la gobernanza compleja. El análisis de este artículo no está confinado a los supuestos de la teoría realista, si bien hay algunos enfoques tales como la interdependencia o el institucionalismo que brindan concepciones más sofisticadas, y el constructivismo provee contribuciones relevantes al abordar la política internacional como una realidad socialmente construida. Hurrell va más allá con su teoría de la gobernanza compleja al avanzar hacia una comprensión más profunda y acabada que amplía el horizonte teórico al considerar que las dinámicas en la política internacional presentan diversas alternativas para su ejercicio y construcción, como se verá a continuación.

Andrew Hurrell está vinculado con la escuela inglesa, que se diferencia del realis$\mathrm{mo} /$ neorrealismo, el neoliberalismo y el constructivismo (2002, pp. vii-xxiii). En efecto, el profesor Hedley Bull fue su guía intelectual, quien reconoció "la existencia de una sociedad internacional conformada por un grupo de Estados conscientes de ciertos intereses y valores comunes, que forman una sociedad en el sentido de que se consideran vinculados por las mismas reglas en sus interacciones y comparten el funcionamiento de instituciones comunes" (Bull, 1977, p. 13). De esta forma, según Ibáńez Muńoz (2010), Hurrell comparte con la escuela inglesa la manera como concibe la existencia de una sociedad internacional en la que actores como las instituciones y organizaciones internacionales juegan un rol determinante $y$, por ende, han logrado disipar los niveles de anarquía que caracterizaban al sistema internacional desde un enfoque realista.

Sin embargo, la sociedad internacional descrita por Hurrell amplía esta definición enfocándose en la manera como los Estados interactúan con una serie de actores sociales, como la sociedad civil, y el papel de dichos actores en la coordinación descentralizada de la sociedad sin que estos tengan que cumplir con las reglas establecidas para los Estados (2007, pp. 95-96). Este autor afirma que la sociedad internacional se ha transformado de manera rápida, dejando de ser Estado-céntrica para convertirse en una sociedad mucho más amplia y compleja. El resultado es lo que llama gobernanza compleja. Para Santos Carrillo (2010), lo diferencian de sus predecesores de la escuela inglesa su énfasis en la normativa del orden global y su punto de partida, según el cual el orden internacional y la gobernanza nunca son procesos políticamente neutrales.

Asimismo, las siguientes propuestas separan a Hurrell de la escuela inglesa y hacen interesante su pensamiento como enfoque teórico de este artículo: en primer lugar, ofrece un orden político no basado en la autoridad jerárquica porque el término "gobernanza" describe la interacción horizontal entre los Estados y otros actores a través de redes y comunidades políticas por los avances tecnológicos en los campos de transporte, comunicación y la transmisión de información; en segundo lugar, el orden internacional ya no está amparado 
por los objetivos comunes mínimos de los Estados, sino por una densa red de normas y una diversidad de actores, con una filosofía similar a la del institucionalismo; en tercer lugar, hay que tener en cuenta a las continuas prácticas sociales en las relaciones internacionales. Solo contemplando estas alternativas es posible hacer inteligible el reto ecológico que es sumamente complejo y problemático.

Hurrell coincide con autores como Celestino del Arenal, quien considera que la sociedad internacional se encuentra en proceso de mutación entre un mundo de Estados que continúa anclado en el viejo dogma de la soberanía nacional y un mundo complejo, global e interdependiente en el que no existen fronteras (Del Arenal, 1990). Hurrell considera, respecto de las funciones del Estado, que son sustituibles y pueden ser asumidas por agencias externas, compañías privadas o un amplio espectro de actores transnacionales. Pero hay quienes impugnan al pensamiento de Hurrell sobre que el estatus y la soberanía de los Estados son elementos que desaparecerían con el tiempo. Por ejemplo, Roberto Rusell (2010 p. 92) documenta la forma en que Stephen Krasner, en su libro Soberania. Hipocresía organizada, demuestra cómo a finales de la década de los noventa, el Estado-nación había vivido un proceso de transformación que lo hacía menos interdependiente y por ello se había fortalecido.

Siguiendo a Roberto Russell (2010) se observa que las características de la gobernanza compleja y su forma de entender las relaciones de poder y cooperación entre actores ha despertado fuertes controversias teóricas, en particular entre las teorías realistas y liberales. La polémica, según Russell, ha girado en torno al cuestionamiento de los Estados como actores protagónicos del sistema internacional, la integralidad del Estado (concepción realista de unidad), y la racionalidad del actor (p. 90).

Por otra parte, desde la óptica de Hurrell (2007), los neoliberales tienen una visión reducida respeto a la gobernanza global, al limitar su entendimiento a la provisión de bienes públicos y a la resolución de problemas de acción colectiva, los cuales no tienen en cuenta el problema de las diversidades de poder, valores e identidades. Desde otra perspectiva, subraya que para los realistas y neorrealistas (institucionalistas), la globalización no es vista como una amenaza en el sistema internacional y, por ende, no puede delimitar el futuro del Estadonación. Por el contrario, la gobernanza es un proceso que los Estados permiten y que es impulsado por ellos (pp. 92-93).

Josep Ibáñez Muñoz afirma que la forma de entender la gobernanza compleja de Hurrell se ajustaba de una manera más simple al enfoque de la teoría transnacionalista, con autores como Robert O. Keohane y Joseph S. Nye, quienes a finales de los años setenta afirmaron que la política y la economía internacional estaban viviendo profundas transformaciones, que eran resultado de las interacciones que establecían entre sí una gran diversidad de actores -estatales y no estatales- en el marco de la denominada "interdependencia compleja" (2010, p. 333).

La relevancia de la contribución intelectual de Andrew Hurrell también ha sido destacada por varios autores. Thomas G. Weiss y Rorden Wilkinson (2014, p. 20) argumentan la importancia del estudio de gobernanza global para las relaciones internacionales, 
notando la contribución de Hurrell como uno de los pocos autores modernos enfocados en el orden global. A la vez, autores interesados en el estudio de ciertas instituciones, como la promoción de los derechos humanos en la onU, hacen referencia a la pertinencia de la gobernanza compleja de Hurrell (Pegram, 2015, p. 598).

Teniendo en cuenta los aportes de $\mathrm{Hu}$ rrell, se toma como estrategia teórica de este artículo su enfoque, el cual es pertinente porque permite examinar el papel de la sociedad civil para promover la gobernanza compleja por medio de cuatro actividades. La primera es participar en el proceso formal de la creación y el desarrollo de normas, y el establecimiento de estándares. La segunda es participar en el proceso social de la divulgación de nuevas normas y su inclusión en la agenda internacional. La tercera es aprovechar su conocimiento de las funciones internas de los organismos internacionales y su habilidad de involucrarse en puntos vitales. Y la cuarta es participar en actividades de gobernanza como la verificación de cumplimiento de compromisos (Hurrell, 2007, p. 101).

En este orden de ideas, el enfoque teórico del artículo se basa en las tres formas de analizar la contribución de la sociedad civil en la transformación de la sociedad internacional hacia una sociedad caracterizada por gobernanza compleja según Hurrell (2007). La primera se enfoca en las herramientas de influencia que utiliza, siendo estas: la habilidad de aprovechar el conocimiento científico e ideas causales, la capacidad de explotar plataformas institucionales, el acceso a los Estados a través de redes y comunidades políticas, y la habilidad de participar en actos políticos simbólicos que influyen en la interpretación de asuntos políticos. La segunda se enfoca no tanto en su influencia, sino más bien en términos de grandes cambios en la conciencia y la creación de nuevas identidades políticas. Y le tercera hace referencia al cambio de foco de lo empírico a lo normativo (pp. 102-104).

Para finalizar, se destaca que Hurrell cita expresamente a Margarita Keck y Katherine Sikkink dentro del argumento sobre las formas en que los actores de la sociedad civil pueden influir en las relaciones interestatales, y para referirse a los recursos que estos actores pueden emplear. Dice que estas autoras

...han destacado de manera influyente los papeles de las redes transnacionales de advocacy ${ }^{3}$. Especialmente en relación con asuntos como los derechos humanos y el medio ambiente, esas redes incluyen no solamente las organizaciones no gubernamentales (ONG), sino también iglesias, los medios y las fundaciones caritativas (Hurrell, 2007, p. 102).

Estas autoras coinciden además con $\mathrm{Hu}$ rrell en destacar la importancia de los valores y las ideas, en creer que los individuos pueden lograr impactos, y en el uso creativo de la información (Keck y Sikkink, 1998, pos. 137).

En estas latitudes, tratándose de la participación de la sociedad civil, vale la pena mencionar a Erli Margarita Marín Aranguren,

3 Para abogar (Marín, 2011, p. 25). 
autora de un conjunto de publicaciones dentro de las que se destaca el libro La sociedad civil: un tabú en Colombia. Marín hace una labor importante en presentar la composición, las estrategias y los escenarios de las organizaciones de la sociedad civil (osc), destacando el carácter dinámico de dichas organizaciones cuando escribe sobre sus ámbitos de interacción. Los escenarios en que actúan las osc incluyen las redes, los movimientos sociales y los medios de comunicación. Las redes "pueden ser locales, globales o glocales y, además, pueden ser físicas o virtuales" (2011, p. 29).

Marín ofrece herramientas que han sido tenidas en cuenta en el presente análisis, tales los "reeditores sociales", es decir, "sujetos que cuentan con credibilidad y reinterpretan mensajes, en función de un propósito temático colectivo" (2011, p. 41). Las osc necesitan a los reeditores sociales para construir puntos de vista que puedan ser escuchados por los tomadores de decisión. También se tuvo en cuenta que los cambios tecnológicos juegan un papel importante. El internet conecta las osC con sus contrapartes en diferentes lugares, facilitando la creación de redes transnacionales. La distribución de información está facilitada por el internet a través de herramientas como YouTube, Twitter y blogs. La autora dice que "el ciberespacio proporciona nuevos repertorios de acción colectiva. La típica recogida de firmas o el envío de cartas de protesta a los gobiernos o a las empresas, toman una nueva dimensión” (pp. 45-46).

\section{RESULTADOS DE LA INVESTIGACIÓN}

A continuación se presentan los resultados del análisis de la participación de tres actores de la sociedad civil en la Cumbre de la Tierra de 1992.

\section{El Consejo Internacional para la Ciencia (ICSU)}

El ICsu es lo que Peter Willetts llama una organización híbrida de agencias gubernamentales y organizaciones no gubernamentales especialistas. El sistema de toma de decisiones que maneja el ICSU es unicameral ${ }^{4}$. Durante el periodo de estudio estuvo compuesto por miembros asociados a agencias nacionales (representando 77 países) y 20 miembros no gubernamentales (comunidades científicas), los cuales fueron organizaciones no gubernamentales internacionales dedicadas a ciertos campos de estudio (Our Members, 2016; ICsU 2016).

En 1969, el ICSU estableció el Comité científico sobre problemas del medio ambiente (scope por sus siglas en inglés) (Willetts, 2011, p. 76), que fue importante en la definición del concepto de cambio climático. Este comité también trabajó para establecer relaciones políticas entre el ICsu y la Organización Meteorológica Mundial (OMM) con el fin de promover la investigación científica. En 1979, el Programa Mundial sobre el Clima fue convocado con el fin de asesorar el impacto del cambio climático (Kates, 1985). Allí el ICsu y el Programa de las Naciones Unidas para el

\footnotetext{
4 Las decisiones se toman por medio de un voto donde cada miembro tiene un voto de igual valor. Gana la posición con la mayoría (sin importar si son organizaciones no gubernamentales) (Willetts, 2011, p. 73).
} 
Medio Ambiente (PNUMA), la omm y unesco analizaron el conocimiento científico sobre cambio climático.

El Programa condujo al Congreso de Villach en Austria en 1985, organizado por la OMM, el PNUMA y el ICSU para "Evaluar el papel de dióxido de carbono y otros gases de efecto invernadero en las variaciones del clima y los impactos asociados" (International Collaborations and Partnerships on Climate Change, 2016, 25 de enero). Los resultados del congreso afirmaron el impacto humano sobre el cambio climático, pronosticando que en el siglo XXI el aumento de la temperatura sería superior a cualquier época de la historia humana. La Comisión Brundtland fue consciente de la importancia del Congreso de Villach e hizo referencia directa a sus resultados en el Informe Brundtland (onu, 1987, par. 24).

Es necesario entender la relevancia de la Comisión Brundtland en la formación de la agenda de la Cumbre de la Tierra. Sin embrago, en 1987 esta Comisión se disolvió y fue reemplazada por el Centro para Nuestro Futuro Común. Este centro tuvo el propósito de promover el Informe Brundtland en la Cumbre de la Tierra; una de las herramientas que utilizó para la promoción fue el Comité Internacional de Facilitación (CIF) compuesto por un sector independiente (los actores de la sociedad civil y las corporaciones).
También vale la pena destacar que el Congreso de Villach influenció la creación del Grupo Intergubernamental de Expertos sobre el Cambio Climático (IPCC por sus siglas en inglés), una comunidad internacional de especialistas que guía la agenda internacional sobre el cambio climático (International Collaborations and Partnerships on Climate Change, 2016). Este grupo fue creado en 1988 para proporcionar una mirada clara y científica del estado actual del conocimiento sobre el cambio climático en el mundo (Organization, 2016, 25 de enero). El primer informe del IPCC en 1990 resaltó la amenaza del cambio climático, y tuvo un papel decisivo en la creación de la Comisión Marco de las Naciones Unidas sobre el Cambio Climático (CMNUCC), una de las instituciones legalmente vinculantes que surgió de la Cumbre de la Tierra (IPCC, 2016).

\section{La Unión Internacional para la Conservación de la Naturaleza y los Recursos Naturales (UICN)}

La UICN es otro ejemplo de una organización híbrida con un sistema bicameral ${ }^{6}$ de Estados y organizaciones no gubernamentales científicas. Al igual que el ICSU, la UiCN ha sido destacada por su contribución científica, siendo pionera del concepto de desarrollo sostenible y promotora del CDB.

\footnotetext{
5 Traducido del inglés de: https:/www.wmo.int/pages/themes/climate/international_background.php

6 Un sistema bicameral trata los votos de los miembros gubernamentales diferente a los miembros no gubernamentales. En el caso de la IUCN, tienen tres categorías de votos. Categoría A son los miembros gubernamentales, compuesta por 86 Estados y 120 agencias gubernamentales. Categoría B está compuesta por 812 ong nacionales y 90 ong internacionales. Categoría C está compuesta por 33 ong más pequeñas e individuos que se especializan en algún campo. Solo las categorías A y B tienen el derecho al voto (Willetts, 2011, pp. 78 y 79).
} 
Según Thomas Princen y Matthias Finger (1994, p. 190), el concepto "desarrollo sostenible" apareció por primera vez en el año 1980 en el documento publicado por la UICN en colaboración con el PNUMA, la Organización para la Agricultura y la Alimentación (OAA), UNESCO, el World Wildlife Fund (WwF), etc., llamado World Conservation Strategy: Living Resource Conservation for Sustainable Development, conocida como la Estrategia Mundial sobre la Conservación. Dicho documento se enfocó en proponer estrategias a los gobiernos para lograr el desarrollo sostenible, basado fuertemente en la conservación de los recursos naturales para las generaciones futuras (IUCN, 1980, sec. 20). Según Sélim Louafi, antes de su publicación no existía ningún vínculo entre conservación del medio ambiente el desarrollo (2007, p. 114). Después, con el Informe Brundtland, el concepto fue incorporado como un asunto central en las negociaciones de la Cumbre de la Tierra.

Una de las influencias más notables de la Estrategia Mundial sobre la Conservación fue el Convenio sobre la Diversidad Biológica $(\mathrm{CDB})$, instrumento legalmente vinculante que surgió en la Cumbre de la Tierra. Según Louafi, los objetivos descritos en la Estrategia Mundial sobre la Conservación son los mismos (aunque con otras palabras) que aparecen en el CDB.

La UICN mostró su habilidad de participar en múltiples niveles, realizando la investigación científica, participando en su implementación política y poniendo en práctica las actividades de mitigación de problemas medioambientales. Dos mecanismos que utiliza para facilitar sus relaciones institucionales son el Grupo para la Conservación de Ecosistemas, que gestionó sus relaciones con varias agencias de la ONU, y el
Centro para el Monitoreo de la Conservación Mundial, que sirve para asesorar la agenda de las Conferencias de las Partes del CDB. A la vez, el Centro de la Conservación para el Desarrollo sirvió para posicionar a la UICN de forma que pudo participar en el diseño de proyectos de desarrollo.

Fuera de su contribución científica, la UICN y el ICSU participaron directamente en actividades políticas claves para el surgimiento de la Cumbre de la Tierra. Ambos actores estuvieron invitados a la revisión del Congreso de Estocolmo en 1982 en Nairobi, lo cual inspiró el estableciendo la Comisión Brundtland. También participaron directamente en el Centro para Nuestro Futuro Común con el fin de promocionar el Informe Brundtland entre los gobiernos e individuos en apoyo de la Cumbre de la Tierra por medio de consultas que ofrecieron a los gobiernos.

El secretario general de la Cumbre de la Tierra consideró que la participación de los actores de la sociedad civil era muy importante para su éxito y decidió aprovechar del conocimiento especializado de varios grupos, pidiéndole a organizaciones como la UICN que proporcionaran consultas sobre temáticas específicas medioambientales a los gobiernos de los Estados miembros de la ONU.

La participación de la UICN en la creación de la Agenda Ya Wananchi: Citizens' Action Plan for the 1990's demuestra la variedad de las estrategias utilizadas para influir en Cumbre de la Tierra. Dicha agenda, liderada por el Environment Liaison Committee International (ELCI), fue el resultado de una negociación de varias organizaciones de la sociedad civil que tomó lugar durante la Cumbre de la Tierra, 
llamado The Roots of our Future. Este produjo un listado de recomendaciones para gobiernos y compromisos de grandes organizaciones como la UiCN (Kingman, 1991).

\section{Asociación para el Progreso de las Comunicaciones (APC)}

La APC se dedica a brindar acceso a la información. La visión de la APC es que "[todas] las personas tengan acceso libre y abierto al internet de manera fácil y a un bajo costo para mejorar sus vidas y hacer del mundo un lugar más justo" (APC, 2016).

En los años anteriores a la Cumbre de la Tierra, la APC fue muy activa en su propósito de extender el acceso a lo que hoy es el internet ${ }^{7}$. En el momento de la Cumbre, sus servicios fueron usados no solamente por los actores de la sociedad civil, sino por los gobiernos y la ONU.

En el año 1987 se formó el primer vínculo transatlántico de computadores, establecido entre PeaceNet/EcoNet en Estados Unidos y GreenNet en Inglaterra. En 1990, la red entre Estados Unidos e Inglaterra se unió para formar la APC, a fin de proveer los servicios de internet para las organizaciones de la sociedad civil (Willetts, 2011, p. 96). Se dio una expansión importante entre 1987 y 1992, incorporando nuevas regiones como América Latina y los países nórdicos. Los expertos de la APC visitaron a los actores de la sociedad civil de ciertos países y regiones para instalar los equipos y entrenar a los individuos encargados. Ciertas personas notables son Mike Jensen, quien trabajó para vincular las redes canadienses, australianas y africanas a la red de APC antes del año 1992, y Carlos Alfonso, de Brasil (el país anfitrión de la Cumbre de la Tierra), quien trabajó con el PNUD, el gobierno italiano y el Instituto Brasileño para dirigir el proyecto de vincularse con la APC. El líder de la APC en esta época fue Robert Pollard, quien logró establecer una relación directa con el secretario general de la Cumbre de la Tierra (Pollard, 1990).

En el año 1990, la APc brindaba sus servicios de comunicación a miles de actores de la sociedad civil de 70 países alrededor del mundo (Gore y Pollard, 1990). Sobre el transcurso de los Comités Preparatorios y la Cumbre de la Tierra, la APC proporcionó conferencias electrónicas (e-conferencias) abiertas a la sociedad civil para debatir los asuntos de la Cumbre, e-conferencias con acceso restringido, e-conferencias solo para lectura donde la Secretaría montó documentos oficiales y entrenamiento en uso de la red (Willetts, 2011, p. 101).

\section{DISCUSIÓN SOBRE LAS ACTIVIDADES DE GOBERNANZA COMPLEJA}

Para discutir sobre el primer objetivo del artículo se empieza por la primera actividad de gobernanza compleja de Hurrell que se analiza, siendo esta la participación de los actores de la sociedad civil en el proceso formal de la creación de normas para establecer estándares

\footnotetext{
7 En esa época fueron una serie de redes (nets) que pudieron combinar para brindar la comunicación a grandes cantidades de usuarios por bajos costos. FidoNet fue el más común y accesible.
} 
y desarrollar normas. Antes de empezar con los tres estudios de caso, se puede observar que la participación de los actores de la sociedad civil fue parte de la estrategia del secretario general del evento, Maurice Strong (Burgiel y Wood, 2012, p. 127). Es así como la acreditación de las organizaciones de la sociedad civil por ECOSOC fue parte del plan del secretario general con el fin de asegurar la inclusión de diferentes actores en las negociaciones.

Esta estrategia constituye una actividad de gobernanza compleja en la medida que se refiere al proceso formal o los procedimientos establecidos institucionalmente para la formación de normas y estándares. Para esto se acreditaron más de 1.400 organizaciones de la sociedad civil después de PrepCom iv en 1992, lo que le permitió a esas organizaciones el acceso formal a los procedimientos de la Cumbre de la Tierra. Peter Willetts hace referencia a la resolución de Ecosoc donde se habla de los derechos que tienen las organizaciones de la sociedad civil que están acreditadas. Todas las organizaciones acreditadas tienen derecho a realizar actividades consultivas, recibir documentos y asistir a ciertas reuniones (Willetts, 2011, p. 193; ONU, 1996). Entonces se puede concluir que la acreditación de las organizaciones de la sociedad civil posibilitó su participación de manera formal en la generación de normas y estándares durante la Cumbre de la Tierra a través de consultas directas con los gobiernos.

En lo que respecta a los tres estudios de caso, la evidencia demostró que en la preparación para la Cumbre de la Tierra, el ICsu y la UICN participaron en el proceso formal de la creación de normas, en concreto en la creación de los conceptos nucleares de las negociacio- nes, cambio climático y desarrollo sostenible, y contribuyeron al desarrollo del CDB.

En efecto, el ICSU participó directamente en el proceso formal de la creación de la CMNUCC, empezando con el Programa Mundial sobre el Clima en 1979 y el Congreso de Villach en 1985. La UICN también participó en el proceso formal de creación del CDB y la Declaración de Rio a través de la Estrategia Mundial para la Conservación en 1980. Se puede argumentar que ambos casos fueron incorporados al proceso formal gracias a su inclusión en el Informe Brundtland (onU, 1987, pars. 22,24 y 28). Este informe promocionó la inserción de los asuntos de desarrollo sostenible, cambio climático y diversidad biológica en la agenda de la Cumbre de la Tierra, resultando en la firma de la Declaración de Rio (con los 27 principios de desarrollo sostenible), de la CMNUCC y el CDB.

La segunda actividad de gobernanza compleja que Hurrell destaca es la participación de los actores de la sociedad civil en el proceso social de la aparición de nuevas normas y su inclusión en la agenda internacional. Cuando Hurrell se refiere al proceso social, se trata de las actividades realizadas por los actores de la sociedad civil para promocionar una perspectiva o idea.

Según la evidencia encontrada, esta fue la única actividad de gobernanza compleja que adelantó la APC. Sin embargo, fue muy importante porque sus contribuciones cambiaron para siempre las herramientas de comunicación y transparencia en eventos de negociaciones internacionales. La importancia de la APC se basa en el éxito que tuvo en la promoción de sus valores y en el hecho de que gracias a sus 
esfuerzos, numerosas organizaciones pudieron participar en el proceso social de la aparición de nuevas normas. La norma que la APC buscó socializar fue una de transparencia y comunicación instantánea a través de correos electrónicos que pudieran ser recibidos desde cualquier parte del mundo con acceso a una red formada por una parte de la APC o acceso a una de las e-conferencias mencionadas.

Aunque en el momento de la Cumbre de la Tierra la norma de comunicación no estaba establecida formalmente, Peter Willetts argumenta que gracias a la APC, la Agenda 21 (otro documento ratificado al final de la Cumbre) promueve la comunicación a través de internet (Willetts, 2011, p. 104; Agenda 21, párr. 40.25). El argumento de Willetts destaca la importancia de la APC, no solo como promotora de sus valores, sino como un "reeditor social" para la promoción de valores por parte de miles de otros actores de la sociedad civil. Se encuentra la discusión de la APC como un reeditor social basado en la definición de Marín $^{8}$. Para enfatizar la importancia de la APC se puede mirar el ejemplo del Instituto Internacional para el Desarrollo Sostenible (IISD por sus siglas en inglés) que se aprovechó de las redes de la APC para convertirse también en un reeditor social. El Earth Summit Bulletin', producido por este grupo, informó a todo tipo de actores presentes en la Cumbre de la Tierra sobre temas relevantes.
Basado en estas evidencias, es necesario reconocer, primero, que la APC realizó una actividad de gobernanza compleja: la participación en el proceso social de la aparición de nuevas normas y su inclusión en la agenda internacional, y segundo, que el acceso a los servicios de la APC permitió que numerosos actores de la sociedad civil realizaran actividades de gobernanza compleja. La APC fue pionera en el uso de tecnologías para avances en comunicación.

Actores como la APC y el IISD utilizan un importante elemento de análisis destacado por Keck y Sikkink: la política de información. Primero es necesario mirar a la APC como una red transnacional de advocacy. Estas redes están caracterizadas por la promoción de ideas y valores, la creencia de que los individuos pueden lograr impactos, y el uso creativo de la información. Los valores promocionados por la APC son la comunicación, la transparencia y el acceso a la información. Su creencia en que los individuos pueden lograr impactos se refleja en su entrenamiento de individuos y organizaciones en el uso de su servicio, fomentando la democracia de ideas. Y el uso creativo de la información se refleja en su uso de las varias versiones de las e-conferencias.

Se puede argumentar que durante la Cumbre de la Tierra, la APC utilizó la estrategia de políticas de información. La generación de información políticamente relevante se puede ver en la publicación de las e-conferencias. La capacidad de la APC de dirigir la información

\footnotetext{
8 "Sujetos que cuentan con credibilidad y reinterpretan mensajes, en función de un propósito temático colectivo" (Marín, 2011, p. 41).

$9 \quad$ Hoy en día el Earth Negotiations Bulletin.
} 
hacia donde genera el mayor impacto se manifiesta en el hecho de que la APC y sus e-conferencias fueron utilizadas por las delegaciones gubernamentales y la Cumbre de la Tierra, lo cual indica un acceso amplio de actores en todos los niveles de la Cumbre.

La tercera actividad de gobernanza compleja que se analiza es también tomada de Andrew Hurrell y se trata de la verificación del cumplimiento de compromisos asumidos por los actores. Se plantea que el ICSU y la UICN hicieron y aún hacen uso de esta actividad de gobernanza compleja. Ambos actores realizan el monitoreo en una variedad de sectores medioambientales. Una estrategia importante para ellos siempre ha sido la evaluación de los efectos de las actividades humanas sobre el medio ambiente. SCOPE, el grupo creado por el ICSU, produjo informes científicos cada año desde 1969 hasta el $2000^{10}$. La UICN realiza actividades similares con el World Conservation Magazine y publica varios libros anualmente. Estos casos son especiales porque en lugar de verificar el cumplimiento a través del seguimiento de las actividades de los gobiernos, realizan verificación cuantitativa de qué tan eficaces han sido las estrategias tomadas por la comunidad internacional para mitigar las amenazas medioambientales. Las publicaciones que producen evalúan el estado del medio ambiente en las áreas temáticas que ellos manejan. Se puede concluir, entonces, que el ICSU y la UICN realizan verificación de cumplimiento con los deberes de protección medioambiental a través de la investigación científica.
En términos de la verificación del cumplimiento, vale la pena resaltar el liderazgo que los dos grupos han guiado en la creación de varios mecanismos para el asesoramiento de los avances en temas del cambio climático y la diversidad biológica. Peter Willetts destaca el impacto del ICsu en el diseño del Panel Intergubernamental de Cambio Climático (IPCC), el cual asesora las políticas sobre el cambio climático. De la misma manera, la UICN contribuyó a la creación del Centro para el Monitoreo de la Conservación Mundial (wCMC por sus siglas en inglés), que asesora avances en la política para la protección de la diversidad biológica y apoya los congresos del CDB. Estas evidencias son interesantes porque no necesariamente representan situaciones en que los actores de la sociedad civil realizan seguimiento de actividades de los Estados para verificar el cumplimiento de compromisos, pero representan contribuciones importantes en la creación de mecanismos institucionales para la verificación del cumplimiento de compromisos. El papel que jugaron el ICSU y la UICN en la formación del IPCC y la wCMC demuestra el reconocimiento de la necesidad de la verificación del cumplimiento de los compromisos por parte de ambos actores de la sociedad civil.

La actividad de verificación del cumplimiento de compromisos es una herramienta muy importante para los actores de la sociedad civil que se especializan en un área científica. La UICN y el ICSU son actores conformados por especialistas gubernamentales y no gubernamentales alrededor del mundo, interesados

10 La biblioteca en línea se encuentra en la siguiente página web: http://www.scopenvironment.org/downloadpubs/indexpub.html 
en promover sus causas e influir la política internacional. Su estructura les permite hacer análisis de varios asuntos relacionados con el medio ambiente de manera que encajan en la mayoría de las regiones del mundo. Los dos realizan seguimiento en general, lo cual es relativamente común entre actores de su índole. También, los dos han logrado liderar la creación de mecanismos de seguimiento y asesoramiento institucionalizados en las normas de la ONU, lo que implica un nivel de sofisticación superior al tipico actor de la sociedad civil.

Se puede argumentar que las actividades descritas arriba son ejemplos de lo que Keck y Sikkink llaman políticas de responsabilidad. Para esto, primero es necesario verificar que el ICSU y la UICN puedan ser considerados redes transnacionales de advocacy. La promoción de ideas y valores se manifiesta en su interés en la protección de ciertas dimensiones del medio ambiente. Su creencia en que los individuos pueden tener impactos no se puede demostrar empíricamente, pero se puede suponer basado en su énfasis en la difusión de información a la gente común para promover sus ideas y valores. Y su uso creativo de la información se refiere a su aplicación de políticas de responsabilidad, lo cual se puede ver en sus estrategias de verificación de cumplimiento.

Recapitulando, se observa que los actores de la sociedad civil destacados en este artículo realizaron tres actividades de la gobernanza compleja en relación con la Cumbre de la Tierra en 1992: la participación en el proceso formal de la creación de normas y el establecimiento de estándares; la participación en el proceso social de la aparición de nuevas normas y su inclusión en la agenda internacional, y la verificación del cumplimiento de compromisos.
La presencia notable de cada actor mencionado en el artículo proporciona evidencia de la existencia de la gobernanza compleja como lo describe Andrew Hurrell en su libro $O n$ Global Order.

\section{DISCUSIÓN SOBRE LAS HERRAMIENTAS DE INFLUENCIA}

Para discutir el segundo objetivo del artículo se procede a analizar las herramientas de influencia en la interpretación de los asuntos políticos utilizadas por los actores de la sociedad civil, según Hurrell, para comprobar si se implementaron esas herramientas en los tres estudios de caso a fin de influir en los asuntos políticos en la Cumbre de la Tierra.

La primera herramienta, la habilidad de aprovechar el conocimiento científico, fue utilizada por el ICSU y la UICN en la preparación de la Cumbre de la Tierra. El ICsu jugó un papel de liderazgo durante el Congreso de Villach. Sus actividades en este congreso representan la aplicación de esta herramienta para ejercer influencia sobre la interpretación del cambio climático. El propósito del Congreso de Villach fue el de informar a los gobiernos sobre las causas y los riesgos del cambio climático por medio de talleres. El estatus del ICsu como un líder en la reunión se debe a su experticia en el estudio del cambio climático. Basado en esta evidencia se puede deducir que la herramienta que utilizó el ICsu en la promoción del cambio climático fue su habilidad para aprovecharse del conocimiento científico.

La UICN también se valió de su conocimiento científico para ejercer influencia sobre 
la interpretación de asuntos políticos, proporcionando el informe que introdujo el concepto de desarrollo sostenible. La Estrategia Mundial sobre la Conservación influyó a la interpretación del concepto de desarrollo sostenible y el Convenio sobre la Diversidad Biológica. En su artículo, "Epistemic community and international governance of biological diversity: A reinterpretation of the role of IUCN", Sélim Louafi argumenta que los objetivos del CDB reflejan los objetivos presentados por la Estrategia Mundial sobre la Conservación. Basado en la evidencia se puede notar que la UICN logró estimular mayor interés en la conservación de la diversidad biológica y la negociación del concepto de desarrollo sostenible gracias a su capacidad de comunicar su conocimiento científico a través de un informe patrocinado por el PNUMA. Por tanto, la UICN aprovechó su conocimiento científico para lograr mayor influencia.

Cuando se habla de los actores expertos que se aprovechan de su conocimiento para influir en la interpretación de asuntos políticos, se coincide con los actores conocidos como comunidades epistémicas ${ }^{11}$. La UICN tiene características de una comunidad epistémica que utiliza la información científica para guiar las decisiones políticas. Los estudios de Louafi han demostrado que la UICN empezó a volverse una comunidad epistémica en 1976 con el programa Conservación para el Desarrollo, que fue creado con la intención de vincular la experticia de la UICN con el trabajo de las agencias de desarrollo (2007, p. 113). Según Louafi, la transformación de la UICN en una comunidad epistémica se debe a tres elementos. El primero es el aumento de involucramiento del mundo científico y el énfasis en el intercambio de información a través de talleres y congresos. El segundo es el apoyo a las organizaciones de la ONU con la creación del Grupo para la Conservación de Ecosistemas. Este grupo tuvo la tarea de coordinar las agencias de la ONU y reforzar la experticia y el liderazgo de la UICN. El tercero es la participación en la implementación de proyectos donde se aplica el conocimiento científico a la práctica. Este se refiere a la creación del Centro de la Conservación para el Desarrollo, lo cual ubicó a la UICN donde podía participar en el diseño de proyectos de desarrollo (p. 116).

El ICSU también tiene características de una comunidad epistémica basado en dos de los tres elementos planteados por Louafi. Primero, el aumento de la participación del mundo científico y el énfasis en el intercambio de la información se vio en el 1979 durante el Programa Mundial sobre el Clima. Segundo, el propósito del programa fue el intercambio de información entre las agencias especializadas. El apoyo a la ONU del ICSU se vio en la creación de SCOPE con el propósito de trabajar con la OMM en la investigación sobre el clima

La segunda herramienta de interés para este análisis es la capacidad de explotar plataformas institucionales para influir en la interpretación de asuntos políticos. En esta

11 Una red de profesionales con reconocida experticia y competencia en un dominio particular y una reivindicación de autoridad al conocimiento relevante para las políticas dentro de ese dominio o asunto (Haas, 1992, p. 4). 
discusión se busca comprobar que los actores de la sociedad civil aprovecharon las plataformas institucionales para promover sus agendas.

Se indaga sobre ocasiones en las que los actores de la sociedad civil tuvieron acceso a las plataformas institucionales. En 1982, el ICSU y la UICN fueron invitados al Congreso de Estocolmo con el objetivo de analizar los avances después del Congreso de las Naciones Unidas sobre el Ambiente Humano. Fue debido a esa reunión que se estableció la Comisión Brundtland, que benefició la promoción de sus agendas en la Cumbre de la Tierra. La Comisión incorporó los asuntos de interés del ICsU y la UICN (haciendo referencia directa a sus investigaciones). La Comisión para Nuestro Futuro Común, después, promovió la agenda del Informe Brundtland para su inclusión en la agenda de la Cumbre de la Tierra. Entonces, de manera indirecta, el ICSU y la UICN aprovecharon la plataforma institucional del Congreso de Estocolmo en 1982 y la Comisión Brundtland en 1987 para promover la inclusión de los asuntos de interés en la agenda de la Cumbre de la Tierra.

Durante la promoción del concepto de cambio climático, el ICsu tuvo acceso a plataformas institucionales, lo cual fue importante para la proyección del cambio climático como un tema de prioridad en el esquema internacional. En 1979, la Organización Mundial de Meteorología convocó el Programa Mundial para el Clima. Fue asistido por el ICSU, el Programa de las Naciones Unidas sobre el Medio Ambiente (PNUMA) y unEsCo. Estos fueron los mismos que asistieron al Congreso de Villach en 1985 con el fin de informar a los gobiernos y la ONU. El acceso a esas plataformas insti- tucionales de la ONU trajo beneficios como el liderazgo y la influencia en el desarrollo de normas y mecanismos institucionales. Peter Willetts destaca la relación entre el liderazgo del ICsu y el establecimiento del IPCC y la CMNUCC, implicando que el ICSU pudo aprovechar las plataformas institucionales para tener una gran influencia en el desarrollo de la agenda internacional sobre el cambio climático (Willetts, 2011, p. 77).

La UICN también aprovechó las plataformas institucionales en la promoción de su experticia sobre el vínculo entre la conservación del ecosistema y el desarrollo sostenible. Como ya se estableció, la UICN se convirtió en una comunidad epistémica durante los años setenta y ochenta. Una gran parte fue la creación del Grupo para la Conservación de los Ecosistemas por el pnuma. Fue a través de ese grupo que la UICN pudo coordinar las agencias especializadas de la ONU y difundir su conocimiento científico (Louafi, 2007, p. 116). Por tanto, la UICN pudo explotar la plataforma del PNUMA para realizar su estrategia de difusión de las agendas que propuso.

La APC también se aprovechó de los vínculos con ciertas plataformas institucionales. Los organizadores de la Cumbre de la Tierra reconocieron la utilidad de los servicios ofrecido por la APC y fomentaron su implementación. Según Peter Willetts, en el documento Agenda 21, que resultó de la Cumbre de la Tierra, la ONU se aproximó a promocionar la APC cuando fomentó el uso de redes electrónicas para facilitar negociaciones intergubernamentales (Willetts, 2011, p. 104; Agenda 21, párr. 40.25). Aceptado lo que plantea Willetts, la relación entre la APC y el secretario general de 
la Cumbre de la Tierra estableció a la APC como una entidad experta en la comunicación a través de internet en 1992.

La tercera herramienta utilizada para influir en la interpretación de asuntos políticos según Hurrell es el acceso a los Estados por medio de redes y comunidades políticas. Los tres actores de la sociedad civil de interés para este artículo pudieron aprovecharse del acceso a los Estados. El ICsU y la IUCN tuvieron (aún tienen) acceso directo a los gobiernos gracias a sus estructuras. Los dos son especiales entre los actores de la sociedad civil porque son híbridos. Entonces, se puede argumentar que son redes o comunidades políticas, y que los miembros no gubernamentales pueden acceder a los miembros gubernamentales a través de la estructura burocrática.

La creación de una red política a través de la unión de varias redes electrónicas fue uno de los objetivos de la APC durante la Cumbre de la Tierra. Las redes que construyó la APC reflejan el argumento de Marín sobre la importancia de la comunicación como una herramienta para los actores de la sociedad civil. Marín indica que los movimientos sociales necesitan a los reeditores sociales. Un reeditor social debe cumplir con tres actividades características: tener credibilidad, reinterpretar mensajes y la habilidad de ser escuchados por los tomadores de decisiones (Marín, 2011, p. 42). La credibilidad es una característica subjetiva, pero dado el éxito que tuvo la APC en expandir sus servicios a distintas regiones y agencias de la ONU, se puede deducir que hubo consenso sobre su credibilidad como reeditor social. Su papel de reinterpretar mensajes cuenta con la incorporación de miles de perspectivas en ciertas e-conferencias. El acceso a los tomadores de decisiones se observa en las conversaciones entre el líder de la APC, Robert Pollard y el secretario general de la Cumbre de la Tierra, Maurice Strong.

Cuando finalizó la Cumbre de la Tierra, la APC había contribuido a la construcción de redes alrededor del mundo, con énfasis en los lugares más vulnerables como África Subsahariana, a través de la incorporación de SangoNet de Sudáfrica (Willetts, 2011, p. 193). Se puede concluir que la unión de redes por parte de la APC creó una comunidad política donde los actores de la sociedad civil pudieron tomar parte de las actividades de la Cumbre de la Tierra

La última herramienta que Hurrell destaca es la habilidad de participar en actos políticos simbólicos. Keck y Sikkink definen las políticas simbólicas como la habilidad de aprovechar los símbolos, las acciones o las historias relevantes para resaltar cierta característica de una situación. La UICN realizó un acto político simbólico cuando participó en la reunión llamada The Roots of Our Future organizada por el ELCI. Se considera un acto político simbólico porque la UICN decidió participar en una reunión paralela y con relativamente poco potencial de impactar la Cumbre de la Tierra de manera sustanciosa. La reunión fue promovida por el ELCI, que se había frustrado gracias al manejo oficial de las organizaciones de la sociedad civil por el cIF. La participación en el evento y la publicación en la Agenda Ya Wananchi no lograron impactar la política, pero comunicaron que los actores de la sociedad civil involucrados estaban comprometidos con la preservación del medio ambiente.

Recapitulando, se observa sobre el análisis de las herramientas disponibles a los actores de 
la sociedad civil, que los tres actores destacados en este artículo fueron exitosos. Los tres tuvieron que acceder a las herramientas disponibles para poder realizar actividades de gobernanza compleja durante la Cumbre de la Tierra.

\section{CONCLUSIÓN}

La principal conclusión de este artículo es que la sociedad civil es un actor relevante para enfrentar el reto ecológico, y contribuye a procesos de gobernanza que pueden transformar el sistema internacional en forma tal que proporcione un marco político viable para el manejo del medio ambiente global. La sociedad civil es un nuevo foco de la lealtad política de las personas, quienes se están identificando con las organizaciones de la sociedad civil que enfrentan los problemas medioambientales.

La hipótesis planteada al iniciar la investigación ha sido confirmada. Los actores de la sociedad civil que conforman los casos de estudio del artículo -el Consejo Internacional para la Ciencia (ICSU), la Unión Internacional para la Conservación de la Naturaleza y los Recursos Naturales (UICN) y la Asociación para el Progreso de las Comunicaciones (APC)- realizaron actividades de gobernanza compleja y utilizaron herramientas definidas por Hurrell. Por medio de dichas actividades y herramientas tuvieron el potencial de influir en la interpretación de asuntos políticos de la Cumbre de la Tierra. Cada uno lo hizo de manera diferente, pero todos dejaron marcadas las negociaciones en términos de procedimientos y resultados. El ICSU y la IUCN se beneficiaron con sus vínculos fuertes con varias agencias de la ONU y con varios gobiernos nacionales, lo que les dio un poder importante. La APC proporcionó una forma innovadora de comunicarse por medio de grandes distancias y, de esta forma, le dio poder a otros actores de la sociedad civil para influir en la interpretación de asuntos políticos.

Según Hurrell, la sociedad civil juega un papel en la creación de nuevas identidades. Esto coincide con lo que Paul Wapner llama "world civic politcs", refiriéndose a la influencia de la sociedad civil en la generación de cambios en la conciencia social. Desde esta perspectiva, se abren nuevos campos de investigación: ¿cómo medir la importancia de los actores de la sociedad civil en la generación y la promoción de cambios de la conciencia social? ¿Cómo se manifiestan esas nuevas identidades en la población?

\section{REFERENCIAS}

APC (2016). Acerca de APC. Recuperado de https://www. apc.org/es/node/93

Bull, H. (1977). Anarchical Society: A Study of Order in World Politics. Houndmills: Macmillan Publishers.

Burgiel, S. y Wood, P. (2012). Witness, architect, detractor: The evolving role of NGOs in international environmental organizations. En The Roads From Rio: Lessons Learned from Twenty Years of Multilateral Environmental Negotiations. New York City: Routlage.

Consejo Económico y Social (1997). Resolutions and Decisions of the Economic and Social Council. New York City: Organización de las Naciones Unidas.

Del Arenal, C. (1990). Introducción a las Relaciones Internacionales. Madrid: Tecnos. 
ESANGO (2016). United Nations Civil Society Participation-Consultative status. Recuperado de http:// esango.un.org/civilsociety/displayConsultativeStatusSearch.do;jsessionid=57FD5A00CEF3FE 47E9C72DA327FBB420? method=search\&ses sionCheck=false

Gore, L. J. y Pollard, R. (1990). Computer Communications \& the 1992 United Nations Conference on Environment and Development. Canadá: Canadian Council for International Cooperation.

Goree, K. (2009). Director's Cut No. 122. Recuperado de http://www.iisd.ca/linkages-update/122/ directors-cut/

Haas, P. M. (1992). Introduction: Epistemic communities and international policy coordination. En Haas, P. M. (ed.). Knowledge, Power and International Policy Coordination. South Carolina: University of South Carolina Press.

Hurrell, A. (1992). El medio ambiente y las relaciones internacionales, una perspectiva mundial. En Medio ambiente y relaciones internacionales. Bogotá: Tercer Mundo Editores.

Hurrell, A. (2002). Foreword. En Bull, H. The Anarchical Society: A Study of Order in Wold Politics (pp. VII-XXIII). New York: Columbia University Press.

Hurrell, A. (2007). On Global Governance: Power, Values, and the Constitution of International Society. New York City: Oxford University Press.

Ibáñez Muñoz, J. (2010). Internet, politica y poder en la sociedad postinternacional. Recuperado de http:// www.ehu.eus/cursosderechointernacionalvitoria/ ponencias/pdf/2010/2010_6.pdf

ICSU (2016). Our Members. Recuperado de http://www. icsu.org/about-icsu/our-members

IPCC (2016). History. Recuperado de http://www.ipcc. ch/organization/organization_history.shtml
International Union for Conservation of Nature and Natural Resources (1980). World Conservation Strategy: Living Resource Conservation for Sustainable Development. New York: IUCN-UNEP-WWF.

Kates, R. et al. (1985). Scope 27: Climate impact assessment. En Scientific Committee on Problems of the Environment. Chennai: M S Swaminathan Research Foundation.

Keck, M. y Sikkink, K. (1998). Activists Beyond Borders (libro electrónico). Ithaca: Cornell University.

Kingman, R. (1991). Agenda Ya Wananchi: Citizens' Action Plan for the 1990's, Nairobi: Wananchi Group.

Louafi, S. (2007). Epistemic community and international governance of biological diversity. En Participation for Sustainability in Trade, Hampshire: Ashgate Publishing Company.

Marín, M. (2011). La sociedad civil: un tabú en Colombia. Bogotá: Universidad Externado de Colombia.

Organización de las Naciones Unidas (1945). Carta de las Naciones Unidas. San Francisco: ONU.

Organización de las Naciones Unidas (1985). Resolución 38/161: Process of preparation of the Environmental Perspective to the Year 2000 and Beyond. New York: Asamblea General.

Organización de las Naciones Unidas (1987). Nuestro Futuro Común. New York: Comisión Brundtland.

Organización de las Naciones Unidas (1989). Resolución 44/228: United Nations Conference on Environment and Development. New York: Asamblea General

Agenda 21. (1992). Recuperada de http://www.un.org/ spanish/esa/sustdev/agenda21/agenda21 toc.ht.

Organización de las Naciones Unidas (1992). Programa 21. Rio de Janeiro: onU. 
Organización de las Naciones Unidas (1996). Resolutions and Decisions of the Economic and Social Council. New York: Asamblea General.

Pegram, T. (2015). Global human rights governance and orchestration: National human rights institutions as intermediaries. European Journal of International Relations, 21, 595-619.

Pollard, R. (1990). United Nations Conference on Environment and Development: Information, Public Participation and Communication System. A Preliminary Proposal and Discussion of the Proposal. Information Habitat.

Princen, T. y Finger, M. (1994). Environmental NGOs in World Politics. London: Routledge.

Rodríguez-Becerra, M. (1994). Crisis ambiental y relaciones internacionales. Bogotá: CEREC:

Russell, R. (2010). El Estado nación y los actores gubernamentales no centrales: Una relación complementaria. En Maira, L. (ed.). La politica internacional subnacional en América Latina. Buenos Aires: Libros del Zorzal.
Santos Carrillo, F. (2010). La integración regional centroamericana y la participación de la sociedad civil. Recuperado de http://www.fundacionetea.org/ media/File/tesis_fsantos.pdf

Serbin, A. (2009). Globalización, regionalismo y sociedad civil. Recuperado de http://www.cries.org/wpcontent/uploads/2010/05/Documentos1.pdf

Weiss, T. y Wilkinson, R. (2014). Global governance to the rescue: Saving international relations? Global Governance: A Review of Multilateralism and International Organizations, 20 (1), 19-36.

Willetts, P. (2011). Non-Governmental Organizations in World Politics: The Construction of Global Governance. London: Routledge.

World Meteorological Organization (2016). International Background. Recuperado de https:// www.wmo.int/pages/themes/climate/international_background.php 